\title{
PROBLEM OF NON-MEASURED POINTS IN SURFACE TEXTURE MEASUREMENTS
}

\author{
Pawel Pawlus ${ }^{1)}$, Rafał Reizer ${ }^{2)}$, Michał Wieczorowski ${ }^{3)}$ \\ 1) Rzeszow University of Technology, Faculty of Mechanical Engineering and Aeronautics, Al. Powstańcow Warszawy 12, 35-959 Rzeszów, \\ Poland (ppawlus@prz.edu.pl) \\ 2) University of Rzeszow, Faculty of Mathematics and Natural Sciences, Centre for Innovative Technologies, Al. Rejtana 16c, \\ 35-959 Rzeszów, Poland (rreizer@ur.edu.pl) \\ 3) Poznan University of Technology, Faculty of Mechanical Engineering and Management, Pl. Marii Sklodowskiej-Curie 5, 60-965 Poznań, \\ Poland $\$ michal.wieczorowski@put.poznan.pl, +48 61665 3567)
}

\begin{abstract}
This work is focused on the issue of non-measured points - one of the most important problems in surface texture measurements using optical methods. The fundamental aim of this research is to analyse errors of surface texture measurements caused by the presence of non-measured points. This study is divided into two parts. In the first part, circles with non-measured points were artificially created on peak portions of measured surfaces. In the second part - the results of measurement by a Talysurf CCI Lite interferometer were analysed. A measurement area of $3.3 \times 3.3 \mathrm{~mm}$ contained $1024 \times 1024$ points. The measurements were performed with different intensity of light. Changes of parameters regarding the analysed errors depended on a surface type. The following parameters are susceptible to errors: skewness $S s k$, areal material ratio $S m r$, as well as the following feature parameters: $S p d, S d a, S d v, S h a$ and $S h v$. Inaccuracies of measurement in valley parts of two-process textures led usually to larger errors of parameter computations compared with deviations in peak portions.
\end{abstract}

Keywords: surface texture measurements, optical method, non-measured points, white light interferometer.

C 2017 Polish Academy of Sciences. All rights reserved

\section{Introduction}

The manufacturing industry moves towards a higher degree of production acumen. Some properties, like materials' contact, sealing, friction, lubricant retention and wear resistance are related to the surface topography. The designer should decide on the number of surface topography parameters that will meet the functional demands of a product. The knowledge about the relationship between the product's surface texture and its function is still incomplete. The process of measuring the surface topography gives quantitative information about it. The surface texture is very important for many practical applications [1-8]. Many roughness measuring instruments give absolute measurement values of surface heights. The profile measurement is usually quick, simple and easy to interpret. However, most surface interactions are areal in nature. The areal (3D) texture parameters [9] are more reliable than the profile (2D) ones. Therefore the 3D measurement became popular. The assessment of surfaces using profiles has been employed since the early 1930's. The measurement systems basically involved the use of a mechanical stylus. There are a lot of factors affecting an uncertainty in surface geometry measurements using the stylus technique. The uncertainty is influenced by various environment, measuring equipment, measured objects, software, as well as tip shapes and sizes [10]. There are other methods for measuring roughness [11-13]. Some kinds of errors are common for various measuring techniques, for example errors caused by an improper digital filtration. It was found that for multi-process (stratified) surfaces having traces of more than one process, the application of a standardised Gaussian filter led to distortion of roughness 
values. Cylinder textures after plateau honing are typical examples of two-process surfaces. Although the fine texture marks fall well within a bandwidth accepted for the cut-off, the scratches do not, since they are too wide. It was recommended that a standard cut-off should be increased to $2.5 \mathrm{~mm}$ for this application [14]. A double Gaussian filter $(R k)$ according to the ISO 13565-2 standard can be also applied [15]. An alternative approach is to use robust Gaussian filters, in which additional weights decreased in the places of valleys or peaks. As a result, textures having deep and wide valleys can be filtered properly [16-19].

The time required to collect real data for areal measurements using stylus instruments can appear excessively long compared with the operating time of the optical techniques [20-23]. This could now become the most important disadvantage of the stylus methods. The optical methods are fast, but they are more sensitive to extraneous effects [24]. Among the optical methods, some of new advanced variants of confocal methods and white light interferometry are the most popular. The confocal optical systems are widely applied in engineering and biology because of very high clarity of obtained images, but when a range is increased the resolution tends to decrease; this factor does not occur when using the interferometric methods. The coherence scanning interferometry extends interferometric techniques to surfaces that are complex in terms of roughness, steps and discontinuities. Additional benefits include an equivalent of autofocus at every point in the field of view [24]. The environment can significantly contribute to measurement errors in the coherence scanning interferometer applications. Sharp edges can cause outliers called spikes in the topographic images [25]. If the light intensity received on a photodetector is too low, the surface height cannot be detected. A part of this image can be corrected using interpolation with an algorithm of determining areas with an ambiguous signal. Non-measured points exist also after the application of confocal optical systems. Non-measured points can result from many different causes, like high steepness of the slopes, surface absorbance or reflectivity. However, it is difficult to find in the accessible technical literature works dealing with the effect of errors caused by nonmeasured points on the distortion of surface texture parameter values. Nevertheless, this problem is one of the most important ones in the surface metrology. These errors may cause a false quality assessment of machined elements.

The fundamental aim of this research is to analyse errors of surface texture measurement of various machined surfaces caused by the presence of non-measured points. Another aim is to select the surface topography parameters that are the most and the least sensitive to the analysed errors.

\section{Materials and methods}

This study is divided into two parts. In the first part, circles with non-measured points were artificially created on peak parts of different measured surfaces, with the same measured areas and sampling intervals in perpendicular directions. The measuring area was $3.3 \times 3.3 \mathrm{~mm}$, the sampling interval in perpendicular directions was $5 \mu \mathrm{m}$. Ten samples with simulated errors were studied. A ratio of non-measured points (NM) was in each case $0.73 \%$.

In the second part of this study the results of measurements by a Talysurf CCI Lite interferometer (produced by Taylor Hobson Ltd) with a $0.01 \mathrm{~nm}$ vertical resolution were analysed. A Nikon $5 \times / 0.13 \mathrm{TI}$ objective was used for all the measurements. The measuring area of $3.3 \times 3.3 \mathrm{~mm}$ contained $1024 \times 1024$ measured points. The measurements were performed at a different intensity of light. The results of measurement with the smallest number of nonmeasured points were the reference (best) data. Fifteen surfaces (one- and two-process, isotropic and anisotropic, random and deterministic) were measured and analysed. 
In both parts, TalyMap Gold version 6.1 software, copyright by Digital Surf, was used for filling in non-measured points. They were replaced by a smooth shape calculated from the neighbour points.

Before computations of parameters, flat surfaces were levelled using a least square plane, whereas curved forms of surfaces were removed using polynomials.

\section{Study of surfaces with artificially created non-measured points}

The aim of this part of research was to analyse the effect of surface type on the errors, because the NM ratio and shape of breaks were the same.

Ten surfaces were analysed; four of them were subjected to a detailed study. Fig. 1 shows examples of the analysed textures. The arrows indicate places where non-measured points are present after filling in. They indicate the effect of non-measured points on surface views. One can see that the corrected part of image is clearly visible on the surface after milling, as shown in Fig 1a, because the circle with non-measured points was located in a surface peak part. The modified circles on honed (Fig. 1b) and polished surfaces (Fig. 1d) are also visible. An anisotropic character of these textures is the most probably reason of visibility of the improved surface parts, because the corrected details are isotropic. However, it is difficult to find a circle with filled in non-measured points on the lapped surface with isolated oil pockets, created by the abrasive jet machining (Fig. 1c). The presence of dimples and an isotropic character of the peak surface part after lapping are the most probable reasons of a small visibility of the error.

a)



c)



b)
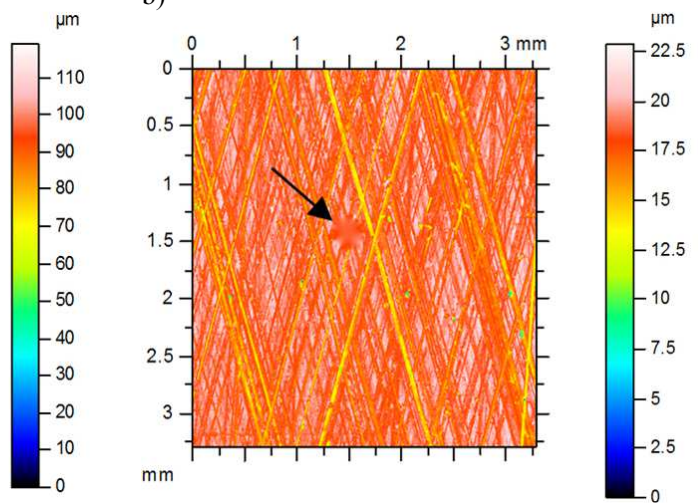

d)

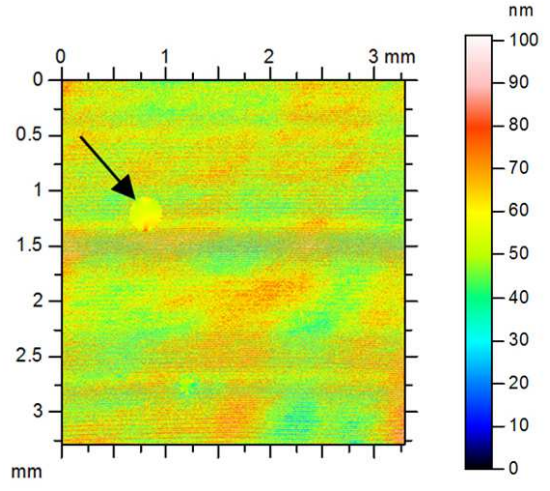

Fig. 1. Contour plots of surface points after milling (a); honing (b); texturing by the abrasive jet machining after initial lapping (c) and polishing (d) after filling in non-measured points. 
Relative changes of the surface topography parameters defined according to the ISO 25178 standard due to disturbances were studied.

Although the error caused a change of the milled surface view (see Fig. 1a), the relative absolute changes of parameter values were not higher than $2 \%$. A different tendency of changes of values for various height parameters was found: for statistical parameters $S a$ (arithmetic mean height) and $S q$ (root mean square height) as well as $S p k$ (reduced summit height) and $S v$ (maximum valley height) they decreased, whereas for $S k$ (core roughness depth) and $S p$ (maximum peak height) parameters the changes increased. They were not higher than $0.7 \%$. Due to a decrease of the $S p k$ parameter as well as $S r 1$ ratio (upper bearing area) values, the $S a 1$ parameter value decreased; its changes were comparatively large (about 1.9\%). The values of both skewness $S s k$ and kurtosis $S k u$ increased. As a consequence of reduced statistical height parameter values, the values of hybrid parameters $S d q$ (root mean square slope) and $S d r$ (developed interfacial area ratio) decreased. The material volume $\mathrm{Vm}$ increased; its changes were the biggest of all the analysed parameters (about $2 \%$ ). Among the feature parameters, $S p d$ (peak density) and $S d a$ (mean hill area) values increased. The spatial parameters were stable. Generally, it was found that the values of parameters related to a peak surface part ( $\mathrm{Sal}$ and $\mathrm{Vm}$ ) changed in the greatest degree.

It is interesting that - from the cylinder liner surface parameters - the closed dale volume $S d v$ value decreased the most (about 4\%) (Fig. 1b). The maximum relative change of other parameter values was $1.7 \%$. From the amplitude parameters only the value of $S q$ decreased, whereas $S p k$ increased. Similarly to the milled surface, the $S s k$ and $S k u$ parameter values increased, whereas those of $S d q$ and $S d r$ decreased. The functional parameter values from $V$ family changed; those of $V m$ increased, whereas $V m c$ (core material volume) and $V v c$ (core void volume) decreased. The feature parameter values $S p d$ and $S d v$ (mean dale volume) decreased while that of $S d a$ increased. Generally, the same tendency of parameter value changes for the milled and honed surfaces was found.

The parameter values of textured surfaces shown in Fig. 1c changed marginally due to the presence of non-measured points. The maximum change of parameter values was $1 \%$ ( $S p d$ and $S d v$ values decreased). The values of the following parameters decreased: $S p k, S a 1, S d r, V v c$ and $S d a$, whereas $S x p$ (extreme peak height) - increased.

The relative errors of parameters for the polished surface shown in Fig. 1d were comparatively large. Those for $S d v$ decreased by about $8 \%$, for $S r 1$ increased by $2.4 \%$ and consequently - for $S a 1$ increased by $3.5 \%$, whereas for $S s k$ and $S d a$ decreased by 3 and $1.6 \%$, respectively. Changes for other parameters were smaller than $1 \%$; for the following parameters they decreased: $S k, S r 2$ (lower bearing area), $S p, S a, S t d$ (texture direction), $S d q, S d r, V v c$ and $S p d$, whereas for the following parameters they increased: $S p k, S a 2$ (oil capacity), $S k u$ and $V m$.

Generally, after analysis of parameter value changes for all ten tested surfaces it was found that deviations of the skewness $S s k$ and kurtosis $S k u$ values occurred (up to 3\%). The values of statistical amplitude parameters $S a$ and $S q$ typically decreased, whereas those of the parameters describing total height $S z, S p$ and $S v$ were more stable. As a result of the amplitude reduction, the values of hybrid parameters also decreased. Since non-measured points were located in the peak regions of analysed surfaces, the values of parameters connected with a peak part: $S r 1, S p k$, sometimes $S k$ and consequently $S a l$ typically changed (up to $4 \%$ ); in most cases the peak density $S p d$ value marginally decreased. The spatial parameters were usually stable; the main directionality described by Str, changed only for the anisotropic one-directional texture (Fig. 1d). Large changes of the feature parameters: $S d v$ and $S d a$ deserve attention. Changes of parameter values depend on a surface type. For example, maximum changes of parameter values shown in Fig. 1c was 1\%; it is difficult to find filled-in non-measured points on this graph. 


\section{Study of surfaces measured in different conditions}

It was found that improper measurement conditions might cause serious inaccuracies in the parameter calculations. High errors of parameter computations occurred even for very small ratios of non-measured points (NM). Fig. 2 presents an example. Non-measured points of a small ratio $(0.0268 \%)$ ) located in a valley surface part caused a considerable increase of roughness height in this part (the $S v$ value increased by more than $20 \%$ ). In addition, the spatial parameter values changed; the autocorrelation length Sal increased by more than $20 \%$, whereas the texture parameter $S t r$ by more than $15 \%$. The peak density decreased by about $25 \%$, whereas the values of feature parameters: $S d a$, $S h a$ (mean hill area), $S d v$ and $S h v$ (mean hill volume) increased by more than $25 \%$. These changes are large, therefore analysis of the surface distortion due to the presence of non-measured points deserves attention. However, the changes of parameter values characterizing the material ratio curve, from the $S k$ family: $S k$, $S p k, S v k$ (reduced valley depth), $S r 1$ and $S r 2$ were smaller than $5 \%$. An increase of the NM ratio from 0.00935 to $0.0268 \%$ caused non-visible changes of the contour plot; therefore it was presented in Fig. 1 only for the reference surface.

From the analysis of several measured surfaces it was found that - due to a growth of relative light intensity from the initial small value - the NM ratio decreased, reached the smallest value and then increased.

a)

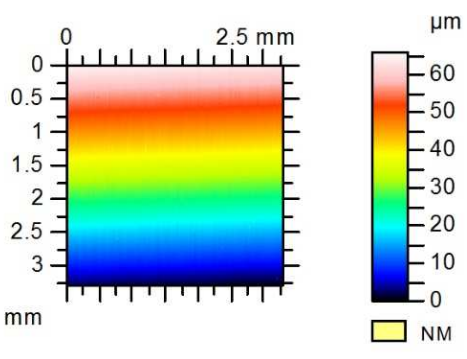

c)

Sk param eters, un filtered.

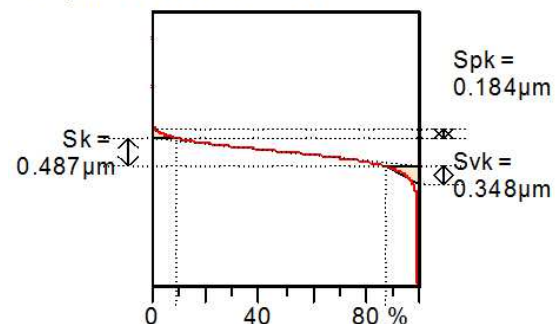

e)

$$
\mathrm{Sr} 1=8.64 \% \quad \mathrm{Sr} 2=87 \%
$$

$$
\begin{gathered}
S v=2.34 \mu \mathrm{m} ; S a l=0.00965 \mathrm{~mm} \\
S t r=0.024 ; S p d=887 / \mathrm{mm}^{2}
\end{gathered}
$$

b)

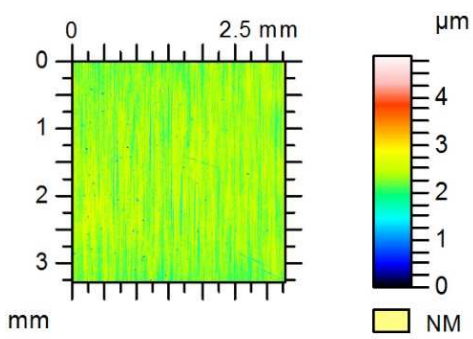

d)

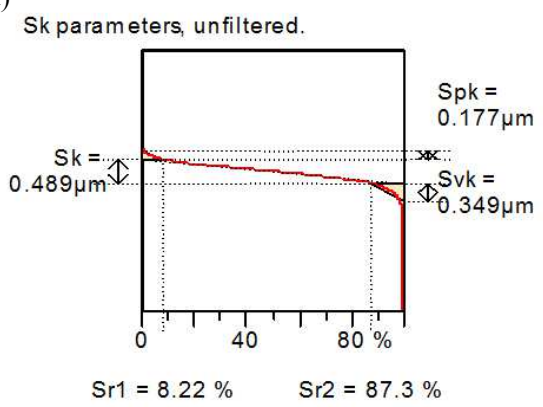

f)

$$
\begin{gathered}
S v=2.81 \mu \mathrm{m} ; S a l=0.0116 \mathrm{~mm} \\
S t r=0.028 ; S p d=669 / \mathrm{mm}^{2}
\end{gathered}
$$

Fig. 2. Contour plots of the polished surface before (a) and after form removal (b) with material ratio curves (c, d) and selected parameters (e, f); graphs a, b, c, e correspond to NM ratio $0.00935 \%$, but d and $\mathrm{f}-0.0268 \%$.

Too small a light intensity caused the presence of non-measured points in a valley region, whereas too high intensity - in a peak surface part. An increase of light intensity caused typically a growth of height, hybrid and functional ( $V$ family) parameter values as well as of oil 
capacity, however for very high NM ratios (larger than 40\%) different tendencies of parameter changes were also possible. The spatial parameter $S a l$ value mainly decreased due to an increase of light intensity. Its changes were higher for the anisotropic surface compared with the isotropic or mixed ones. The variability of $S d q$ parameter is smaller than that of $S d r$. A similar finding was found in other research reports $[22,23]$ and in the first part of the present study.

a)

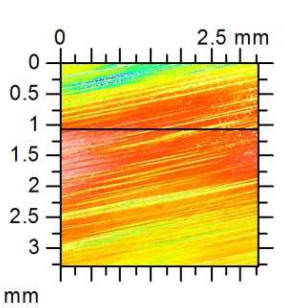

b)





c)

$S q=0.387 \mu \mathrm{m} ; S p=1.13 \mu \mathrm{m} ; S v=2.03 \mu \mathrm{m} ; S s k=-0.76 ; S m r=49.1 \%$

d)

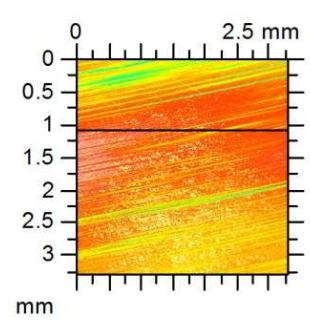

f) e)


$\mu \mathrm{m} \bigwedge$ Length $=3.29 \mathrm{~mm} \mathrm{Pt}=1.57 \mu \mathrm{m}$ Scale $=3 \mu \mathrm{m}$

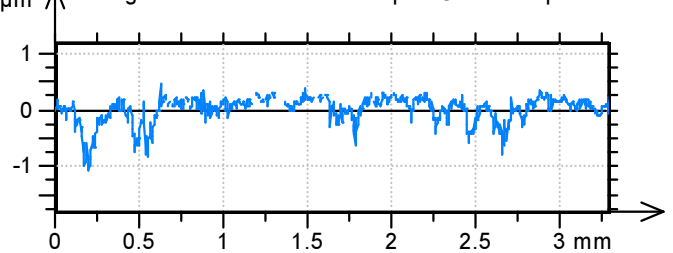

$S q=0.444 \mu \mathrm{m} ; S p=1.47 \mu \mathrm{m} ; S v=3.43 \mu \mathrm{m} ; S s k=-0.78 ; S m r=11.9 \%$

g)

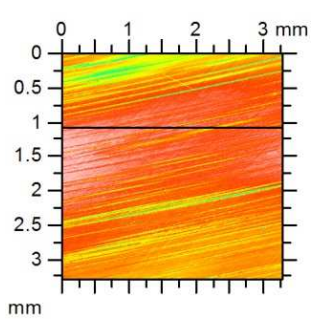

i) h)

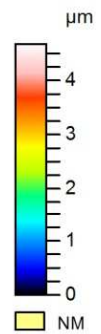

h)



$S q=0.442 \mu \mathrm{m} ; S p=1.27 \mu \mathrm{m} ; S v=3.43 \mu \mathrm{m} ; S s k=-0.81 ; S m r=35.5 \%$

Fig. 3. Contour plots (a, d, g); extracted profiles $(b, e, h)$ and selected parameters $(c, f, i)$ of the polished surface with non-measured points located in a valley part, NM ratio $10.1 \%(a, b, c)$ with non-measured points located in a peak part, NM ratio $9.3 \%$ (d, e, f) with the smallest number of non-measured points, NM ratio $0.1 \%(\mathrm{~g}, \mathrm{~h}, \mathrm{i})$.

The non-measured points caused typically an increase of the $S s k$ parameter and decrease of the $S k u$ parameter values for one-process surfaces. The functional parameters $S m c$ (inverse 
areal material ratio) and especially $S x p$ were more stable than the $S m r$ parameter. A large variation of the $S m r$ parameter value was found in other research reports [26, 27]. Typically, the changes of the values of spatial parameters Str and - especially - Sal were larger than those of height parameters, however a different situation was also possible. The main texture direction Std was constant for the anisotropic surface with the main directionality, contrary to the isotropic textures. It is difficult to find a tendency of changes of the peak density $S p d$, however variation of this parameter value was large. The following feature parameters: $S d a, S d v$, Sha and $S h v$ were also non-stable on the surface. The variability of $S r 2$ material ratio was lower than that of $S r 1$ ratio. Usually, a one-process surface with the highest NM ratio corresponded to maximum changes of parameters.

a)

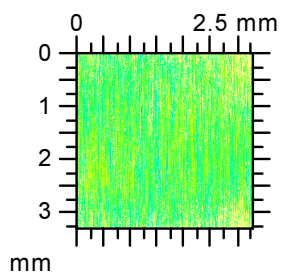

c)



e)

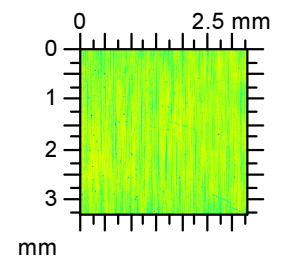

b)

$$
\begin{aligned}
& S q=0.2 \mu \mathrm{m} \\
& S p=2.4 \mu \mathrm{m} \\
& S v=1.53 \mu \mathrm{m} \\
& S s k=-0.42 \\
& \text { Sal }=0.0193 \\
& \text { Str }=0.042
\end{aligned}
$$

d)

$$
\begin{aligned}
& S q=0.24 \mu \mathrm{m} \\
& S p=6.82 \mu \mathrm{m} \\
& S v=1.81 \mu \mathrm{m} \\
& S s k=-0.5 \\
& S a l=0.0245 \\
& S t r=0.107
\end{aligned}
$$

f)

$$
\begin{aligned}
& S q=0.22 \mu \mathrm{m} \\
& S p=2.53 \mu \mathrm{m} \\
& S v=2.34 \mu \mathrm{m} \\
& S s k=-0.68 \\
& S a l=0.0096 \\
& S t r=0.024
\end{aligned}
$$

Fig. 4. Contour plots (a, c, e) and selected parameters (b, d, f) of the polished surface with non-measured points located in a valley part, NM ratio $24.2 \%(a, b)$ with non-measured points located in a peak part, NM ratio $58 \%(\mathrm{c}, \mathrm{d})$ with the smallest number of non-measured points, NM ratio $0.00935 \%(\mathrm{e}, \mathrm{f})$.

Generally, the variability of statistical height parameters $S a, S q$, spatial parameter $S t d$, slope $S d q$, functional parameters from $V$ family as well as material ratio $S r 2$ caused by the presence of non-measured points was relatively small. Large errors existed only for a high NM ratio. However the following parameters are susceptible to errors caused by high NM ratios: skewness $S s k$, functional parameter $S m r$ and the following feature parameters: $S p d, S d a, S d v, S h a$ and Shv.

The changes of parameters depend on a location of non-measured points. Fig. 3 presents an example. A surface of a little asymmetric distribution after polishing was studied. An NM ratio of a texture shown in Figs. $3 \mathrm{a}$ and $3 \mathrm{~b}$ is similar to that presented in Figs. $3 \mathrm{~d}$ and $3 \mathrm{e}$, however one can see from both views of contour plots and extracted profiles that non-measured points 
are located in valley and peak parts, respectively. Due to the presence of non-measured points in a valley part the $S v$ parameter value decreased by about $40 \%$ and $S q$ - by nearly $12 \%$. The presence of non-measured points in a peak surface part led to an increase of the $S p$ parameter value by more than $15 \%$ and a decrease of the $S m r$ value by more than $60 \%$. Due to the presence of non-measured points the $S s k$ parameter value increased. A change of $S a l$ parameter value was smaller than $10 \%$, which was probably related to the fact that a mixed surface (Str about 0.35 ) was analysed.

a)



c)

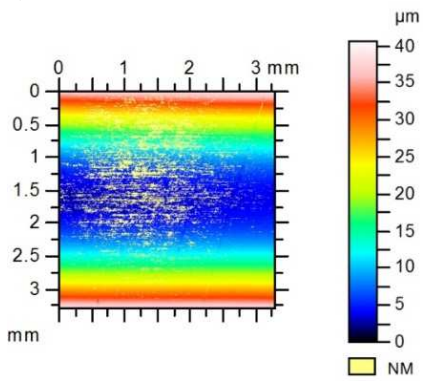

e)

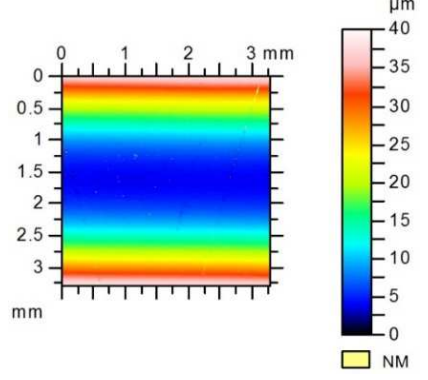

b)



d)

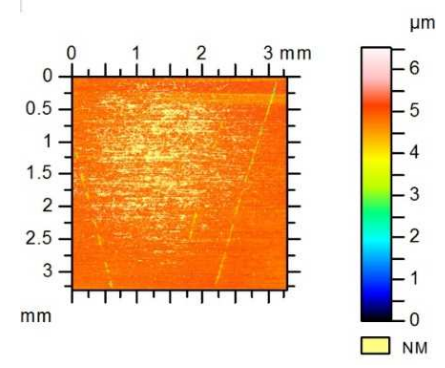

f)



Fig. 5. Contour plots of a worn cylinder surface before (a, c, e) and after form removal (b, d, f) with non-measured points located in a valley region; NM ratio $4.31 \%(\mathrm{a}, \mathrm{b})$, in a peak part; NM ratio $14.4 \%$ (c, d) and with their smallest number; NM ratio $0.102 \%$ (e, f).

The statistical parameters $S q$ and $S a$ are stable for an extremely high number of nonmeasured points. When these points are located in a peak surface part (NM ratio was $58 \%$ - see Figs. 4c, and 4d) the $S q$ parameter value was overestimated by $9 \%$. A similar error was obtained when non-measured points were present in a valley part (NM ratio was $24.2 \%$ - see Figs. $4 \mathrm{a}$ and $4 \mathrm{~b}$ ). Similarly to Fig. 3, the presence of non-measured points in a valley surface part caused large changes of the $S v$ parameter value, but in a peak part - of the $S p$ parameter value. In both cases, errors led to an increase of the parameter values: Sal, Str and Ssk. 
The presented above tendencies are concerned mainly with one-process surfaces. However similar trends were observed also for two-process textures characterized by a negative skewness (usually smaller than -1). An increase of light intensity led in most cases to a decrease of skewness $S s k$ and usually to an increase of kurtosis $S k u$ values. This is reasonable because a small skewness corresponds to a large kurtosis. This tendency was probably caused by the presence of non-measured points in the bottom of valleys (Figs. 3a and $3 \mathrm{~b}$ ).

a)

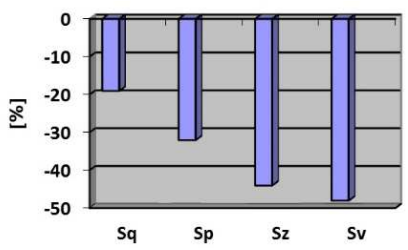

b)

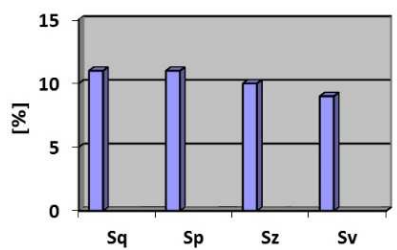

Fig. 6. Relative changes of parameters for the surface presented in Fig. 5a (a) and 5b (b).

a)
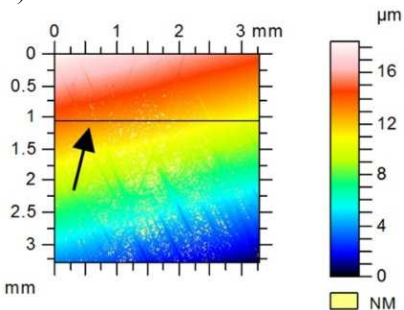

c)



e)

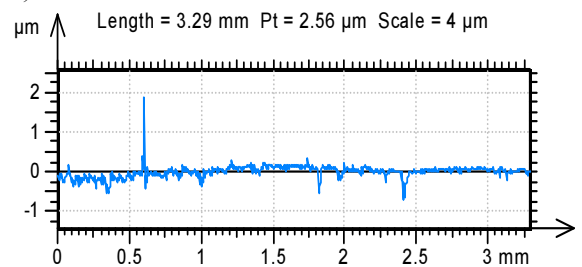

g)

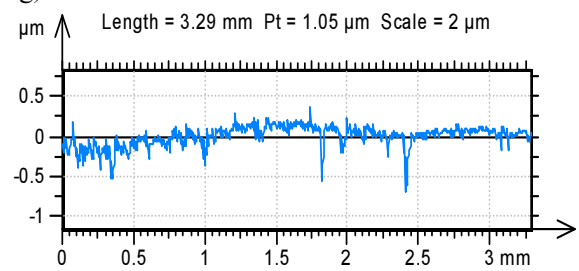

b)

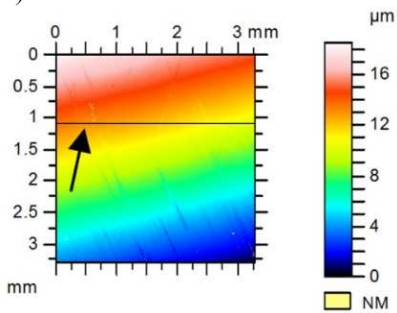

d)



f)

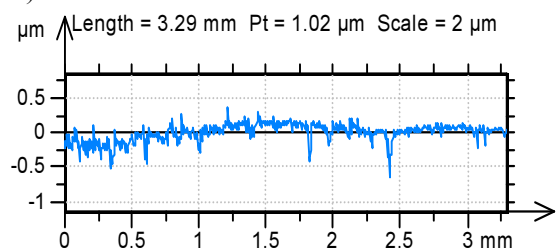

h)



Fig. 7. Contour plots $(a, b)$, extracted profiles before correction - filling in (c, d), after correction of the whole surface $(e, f)$ and after profile correction $(g, h)$; NM ratio $6.68 \%(a, c, e, g)$ and $0.079 \%(b, d, f, h)$. 
For the surface shown in Fig. 3 the change of $S s k$ parameter value is small, but high errors of this parameter calculation can occur for two-process textures. As a result of it a surface machined well looks like a one-process texture and can be classified as a spoilage. An increase of the $S s k$ parameter value is related to a decrease of the $S v k$ parameter value. Errors of twoprocess surface topography parameter estimations are very important, because the functional properties of two-process surfaces are better than those of one-process textures [28-31]. An inaccuracy of a valley part measurement typically led to larger errors of parameter computations compared with a disturbance in a plateau region. For example, the relative errors of parameter values for the surface shown in Figs. 5a and 5b are higher than those for the surface presented in Figs. 5c and 5d, although the NM ratio of the latter is higher. Fig. 6 presents relative changes of typical parameters. Negative changes correspond to underestimation, while positive ones - to overestimation of amplitude parameters. The $S s k$ parameter values of the surface shown in Fig. 5 are: in $5 \mathrm{a}$ and $5 \mathrm{~b}-0.34,5 \mathrm{c}$ and $5 \mathrm{~d}-1.26,5 \mathrm{e}$ and $5 \mathrm{f}-1.25$.

The other tendencies of parameter changes due to the presence of non-measured points found for a one-process surface were also confirmed for two-process textures. The texture direction was typically constant. The feature parameters, especially $S d a, S h a, S d v$ and $S h v$ were nonstable on two-process surfaces.

An incorrect light intensity could lead to the presence of spikes (Fig. 6). However, erasing non-measured points from the whole honed surface can cause an increase of profile height (Fig. $7 \mathrm{c}$ and $7 \mathrm{e}$ ), contrary to a similar correction of profile only (Fig. $7 \mathrm{~g}$ ). When the light intensity was smaller (Fig. 7b, 7d, 7f and 7h) the non-measured points appeared in the same place (see arrows), but without the presence of spikes. The same profile was obtained after correction of the whole surface or profile (see Fig. $7 \mathrm{f}$ and $7 \mathrm{~h}$ ). The $S p$ parameter value for the corrected surface after form removal with a smaller NM ratio was $1.21 \mu \mathrm{m}$, but with a higher one $3.07 \mu \mathrm{m}$. The arrows indicate positions of errors.

\section{Conclusions}

The problem of non-measured points is one of the most important issues in surface texture measurements. The presence of even a small number of non-measured points can cause false estimation of surface texture parameters which can substantially affect the quality assessment of machined elements.

When non-measured points were located in one place of a peak surface part, the values of parameters describing this part changed more than the others. A character of parameter changes depends on a surface type.

An increase of light intensity caused typically an increase of the height, hybrid and functional parameter values; the spatial parameter Sal value mainly decreased. The following parameters are susceptible to errors caused by a high NM ratio: skewness $S s k$, functional parameter $S m r$ and feature parameters: $S p d, S d a, S d v, S h a$ and $S h v$.

For stratified surfaces, an increase of light intensity caused a decrease of skewness $S s k$ and an increase of kurtosis $S k u$ values. These changes can be large. For this type of surface an inaccuracy of measurement in a valley part led usually to larger errors of parameter computations compared with disturbances in a plateau region.

\section{Acknowledgements}

Part of this work was supported by the Polish National Centre of Research and Development (the project contract No. PBS2/A6/20/2013/NCBiR/24/10/2013; "Research and evaluation of reliability of modern methods of surface topography measurements in micro and nano scale"). 


\section{References}

[1] Wieczorowski, M., Mrozek, R., Andrałojć. P. (2010). The use of surface asperities analysis to investigate wear of bodies in contact on example of brake elements. Metrol. Meas. Syst., 17(2), 271-278.

[2] Twardowski, P. (2011). Surface roughness analysis in milling tungsten carbide with CBN cutters. Metrol. Meas. Syst., 18(1), 105-114.

[3] Wojciechowski, S., Twardowski, P., Wieczorowski, M. (2014). Surface texture analysis after ball end milling with various surface inclination of hardened steel. Metrol. Meas. Syst., 21(1), 145-156.

[4] Krolczyk, G.M., Legutko, S. (2014). Experimental analysis by measurement of surface roughness variations in turning process of duplex stainless steel. Metrol. Meas. Syst., 21(4), 759-770.

[5] Lipiński, D., Kacalak, W. (2016). Metrological aspects of abrasive tool active surface topography evaluation. Metrol. Meas. Syst., 23(4), 567-577.

[6] Krolczyk, G.M., Krolczyk, J.B., Maruda, R.W., Legutko, S., Tomaszewski, M. (2016). Metrological Changes in Surface Morphology of High-Strength Steels in Manufacturing Processes. Measurement, 88, $176-185$.

[7] Krolczyk, G.M., Maruda, R.W., Nieslony, P., Wieczorowski, M. (2016). Surface morphology analysis of Duplex Stainless Steel (DSS) in Clean Production using the Power Spectral Density. Measurement, 94, 464-470.

[8] Rosen, B.G., Anderberg, C., Ohlsson, R. (2008). Parameter correlation study of cylinder liner roughness for production and quality control. Proc. of the Institution of Mechanical Engineers, Part B: Journal of Engineering Manufacture, 222, 1475-1487.

[9] Blateyron, F. (2013). The areal field parameters. Leach, R.K. Characterisation of Areal Surface Texture, Springer, 15-43.

[10] Pawlus, P., Wieczorowski, M., Mathia, T. (2014). The errors of stylus methods in surface topography measurements. Zapol.

[11] Murugarajan, A., Samuel, G.L. (2011). Measurement, modelling and evaluation of surface parameter using capacitive-sensor-based measurement system. Metrol. Meas. Syst., 18(3), 403-418.

[12] Zawada-Tomkiewicz, A. (2010). Estimation of surface roughness parameter based on machined surface image. Metrol. Meas. Syst., 17(3), 493-504.

[13] Salazar, F., Belenguer, T., García, T., Ramos, T. (2012). On roughness measurement by angular speckle correlation. Metrol. Meas. Syst., 19(2), 373-380.

[14] Whitehouse, D.J. (1983). Some theoretical aspects of a practical measurement problem in plateau honing. Int. J. Prod. Res., 21(2), 215-221.

[15] Mummery, L. (1990). Surface texture analysis, the handbook. Hommelwerke GmbH.

[16] Brinkman, S., Bodschwinna, H. (2003). Advanced Gaussian filters. Assessment surface topography, Blunt, L., Jiang, X. (eds.). Kogan Page Science, London and Sterling, 62-89.

[17] Brinkman, S., Bodschwinna, H., Lemke, H.W. (2000). Development of a robust Gaussian regression filter for three-dimensional surface analysis. X International Colloquium on Surfaces. Chemnitz, Germany, 122131.

[18] Li, H., Jiang, X., Li, Z. (2004). Robust estimation in gaussian filtering for engineering surface characterization. Precis. Eng., 28(2), 186-193.

[19] Dobrzanski, P., Pawlus, P. (2010). Digital filtering of surface topography: Part II. Applications of robust and valley suppression filters. Precis. Eng., 34 (3), 651-658.

[20] Whitehouse, D.J. (2011). Surface metrology today: complicated, confusing effective? Proc. of the 13th International Conference on Metrology and properties of Engineering Surfaces. Twickenham Stadium, UK, $1-10$.

[21] Leach, R.K. (2011). Optical measurement of surface topography. Springer.

[22] Vorburger, T.V., Rhee, H.G., Renegar, T.B., Song, J.F., Zheng A. (2007). Comparison of optical and stylus methods for measurement of surface texture. Int. J. Adv. Manuf. Tech. 33(1), 110-118. 
[23] De Groot, P. (2015). Principles of interference microscopy for the measurement of surface topography. Adv. Opt. Photonics, 7(1), 1-65.

[24] de Groot, P. (2011). Coherence scanning interferometry. Optical Measurement of Surface Topography, Leach, R. (ed.), Springer-Verlag, Berlin and Heidelberg.

[25] Podulka, P., Pawlus, P., Dobrzański. P., Lenart, A. (2014). Spikes removal in surface measurement. J. Phys. Conf. Ser., 483(1), 012025.

[26] Pawlus, P., Grabon, W., Reizer, R., Gorka, S. (2015). A study of variations of areal parameters on machined surfaces. Surf. Topog.: Metrol. Prop., 3(2), 025003.

[27] Dzierwa, A., Reizer, R., Pawlus, P., Grabon, W. (2014). Variability of areal surface topography parameters due to the change in surface orientation to measurement direction. Scanning, 36(1), 170-183.

[28] Grabon, W., Pawlus, P., Sep, J. (2010). Tribological characteristics of one-process and two-process cylinder liner honed surfaces under reciprocating sliding conditions. Tribol. Int., 43(10), 1882-1892.

[29] Johansson, S., Nilsson, P.H., Ohlsson, R., Anderberg, C., Rosen, B.G. (2008). New cylinder liner surfaces for low oil consumption. Tribol. Int., 41(9-10), 854-859.

[30] Yousfi, M., Mezghani, S., Demirci, I., El Mansori, M. (2015). Smoothness and plateauness contributions to the running-in friction and wear of stratified helical slide and plateau honed cylinder liners. Wear, $332-333,1238-1247$

[31] Mezghani, S., Demirci, I., Yousfi, M., El Mansori, M. (2013). Mutual influence of crosshatch angle and superficial roughness of honed surfaces on friction in ring-pack tribo-system. Tribol. Int., 66, 54-59. 